\title{
Insect Molecular Biology and Ecology
}




\title{
Insect Molecular Biology and Ecology
}

\author{
Editor \\ Klaus H. Hoffmann \\ Animal Ecology I \\ Bayreuth Center of Ecology and Environmental \\ Research (BayCEER) \\ University of Bayreuth \\ Bayreuth \\ Germany
}

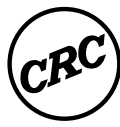

CRC Press

Taylor \& Francis Group

Boca Raton London New York

CRC Press is an imprint of the

Taylor \& Francis Group, an informa business

A SCIENCE PUBLISHERS BOOK 
Cover illustrations reproduced by kind courtesy of Dr. Yuichi Oba, Nagoya University, Nagoya 464-8601, Japan

\section{CRC Press}

Taylor \& Francis Group

6000 Broken Sound Parkway NW, Suite 300

Boca Raton, FL 33487-2742

(c) 2015 by Taylor \& Francis Group, LLC

CRC Press is an imprint of Taylor \& Francis Group, an Informa business

No claim to original U.S. Government works

Printed on acid-free paper

Version Date: 20140814

International Standard Book Number-13: 978-1-4822-3188-5 (Hardback)

This book contains information obtained from authentic and highly regarded sources. Reasonable efforts have been made to publish reliable data and information, but the author and publisher cannot assume responsibility for the validity of all materials or the consequences of their use. The authors and publishers have attempted to trace the copyright holders of all material reproduced in this publication and apologize to copyright holders if permission to publish in this form has not been obtained. If any copyright material has not been acknowledged please write and let us know so we may rectify in any future reprint.

Except as permitted under U.S. Copyright Law, no part of this book may be reprinted, reproduced, transmitted, or utilized in any form by any electronic, mechanical, or other means, now known or hereafter invented, including photocopying, microfilming, and recording, or in any information storage or retrieval system, without written permission from the publishers.

For permission to photocopy or use material electronically from this work, please access www.copyright. com (http://www.copyright.com/) or contact the Copyright Clearance Center, Inc. (CCC), 222 Rosewood Drive, Danvers, MA 01923, 978-750-8400. CCC is a not-for-profit organization that provides licenses and registration for a variety of users. For organizations that have been granted a photocopy license by the CCC, a separate system of payment has been arranged.

Trademark Notice: Product or corporate names may be trademarks or registered trademarks, and are used only for identification and explanation without intent to infringe.

\section{Library of Congress Cataloging-in-Publication Data}

Insect molecular biology and ecology / editor: Klaus H. Hoffmann. pages $\mathrm{cm}$

Includes bibliographical references and index.

ISBN 978-1-4822-3188-5 (hardcover : alk. paper) 1. Insects--Molecular aspects. 2.

Insects--Ecology. I. Hoffmann, Klaus H. (Klaus Hubert), 1946-

QL493.5.I574 2015

595.7--dc23

2014026003

Visit the Taylor \& Francis Web site at

http://www.taylorandfrancis.com

and the CRC Press Web site at

http://www.crcpress.com 


\section{Dedication}

Dedicated to my grand daughters 


\section{Preface}

A major challenge in current entomology is to integrate different levels of organization, from cellular mechanism to function in ecosystem. In the postgenomic era of the 21st century various fields of study have become possible, which use the information of fully sequenced insect genomes (https://www.hgsc.bcm.edu/arthropods/i5k-pilot-project-summary). However, the rapid development of molecular techniques for studying gene functions will revolutionize entomology not only for the insect model organisms, but in general. The majority of these techniques can also be applied if only partial sequence information is available. With these tools, entomologists are able to answer questions in insect biochemistry, physiology, and endocrinology, but also illuminate very complex behavioral and ecological aspects.

When I edited a book on "Environmental Physiology and Biochemistry of Insects" in 1985 for the Springer-Verlag, Berlin, mechanisms of environmental adaptation in growth and development, energy metabolism, or respiration to temperature, oxygen tension, food supply or salt concentrations were in the focus of interest. It was at the time of "Physiological Ecology". About 30 years later, the omics era gives us the opportunity to gain deeper insight into different aspects of insect physiology and environmental adaptation, for example, by overexpression or silencing of candidate genes of interest. When we understand, how physiological processes are regulated and at what time, we will be able to manipulate them, hereby providing attractive potential for practical application, for example, in an ecologically friendly insect pest control.

In 2008, we started with a Master program in "Molecular Ecology" at our University of Bayreuth, which has become very successful during the last 6 years. The Master's program was designed to play a special role in the synergistic cross-linking of the two focal points at our University, "Ecology and Environmental Sciences" and "Molecular Biosciences". The focus of interest is the functions of organisms-and especially of insectsin their environment and the analysis of (bio)chemical interactions in complex ecosystems. "Molecular" should mean not only to study the 
viii Insect Molecular Biology and Ecology

function of macromolecular compounds such as proteins and nucleic acids, but to analyze also the structure and capacity of low molecular weight substances like signal molecules, toxins or drugs. This Master program inspired me to edit the present book on Insect Molecular Biology and Ecology.

The book provides a mix of topical review articles and current research work. In several chapters previously unpublished data are presented showing novel applications for the use of omics technologies in the postgenomic era. The book should prove useful not only to researchers of the Insecta, but also to teachers and graduate students who are interested in understanding the molecular basis of insect functioning in their natural environment.

I acknowledge the support received from the authors who accepted the invitation to write an article on their area of expertise and for delivering the manuscripts in due time. Any success this book may achieve has to be attributed to their efforts.

Bayreuth, July 17, 2014 


\section{Contents}

Dedication

Preface vii

1. Mechanisms of Polyphenism in Insects 1 Stephen M. Rogers

2. Toxins, Defensive Compounds and Drugs from Insects Konrad Dettner

3. Insect Bioluminescence in the Post-Molecular Biology Era Yuichi Oba

4. A Glance on the Role of miRNAs in Insect Life Sassan Asgari and Mazhar Hussain

5. Advances in Insect Physiology and Endocrinology Through Genomics and Peptidomics Ian Orchard and Angela B. Lange

6. Neuropeptide Signaling and RNA Interference Klaus H. Hoffmann, Sandy Weidlich and Franziska Wende

7. Insect Photoperiodism

Shin G. Goto and Hideharu Numata

8. Insects in Winter: Metabolism and Regulation of Cold Hardiness

Kenneth B. Storey and Janet M. Storey

9. Evolutionary Ecology of Insect Immunity

Gerrit Joop and Andreas Vilcinskas

10. The Coleopteran Gut and Targets for Pest Control 
x Insect Molecular Biology and Ecology

11. Trypsin Modulating Oostatic Factor (TMOF) and Insect

Biotechnology

Dov Borovsky

12. RNAi Based Functional Analysis of Biosynthetic Enzymes and Transport Proteins Involved in the Chemical Defense of Juvenile Leaf Beetles

Antje Burse and Wilhelm Boland

13. Silks from Insects-From Natural Diversity to Applications

M. Neuenfeldt and T. Scheibel

Insect Index

Subject Index

Color Plate Section 


\section{7 \\ Insect Photoperiodism}

Shin G. Goto ${ }^{1, *}$ and Hideharu Numata ${ }^{2}$

\section{Seasonal Schedules and the Life History in Insects}

The Earth's rotation on its own axis gives rise to daily changes in light and temperature, while the tilt of the Earth relative to its plane of rotation about the sun causes annual changes in light and temperature and, consequently, the changing of seasons. The length of day and its rate of change are functions of latitude; at higher latitudes, days are longer and day length increases more rapidly than in areas at lower latitudes after the spring equinox. Conversely, at higher latitudes, days are shorter and day length decreases more rapidly as compared to lower latitudes after the autumn equinox. Colder climates are typically associated with higher latitudes, which restrict the duration of seasons that are ideal for growth and reproduction even with longer days. Insects have responded to these geographic variations in climate by evolving appropriate modifications to their photoperiodism.

Photoperiodism is an adaptive, seasonal timing system that enables organisms to coordinate their development and physiology to annual changes in the environment using day length (photoperiod) as a cue (Nelson et al. 2010). Insects must concentrate their reproductive efforts into seasons that favor the development and survival of their offspring,

\footnotetext{
${ }^{1}$ Graduate School of Science, Osaka City University, Sumiyoshi, Osaka 558-8585, Japan. Email: shingoto@sci.osaka-cu.ac.jp

${ }^{2}$ Graduate School of Science, Kyoto University, Sakyo, Kyoto 606-8502, Japan.

Email: numata@ethol.zool.kyoto-u.ac.jp

* Corresponding author
} 


\section{Insect Molecular Biology and Ecology}

and other activities, such as growth, migration, and diapause, must also be timed relative to seasonal abiotic and biotic environmental changes. Photoperiodism was first demonstrated in plants nearly a century ago (Garner and Allard 1920). Shortly thereafter, the first published report of photoperiodism in animals described the switch to oviparity in response to short days in the female strawberry root aphid Aphis forbesi Weed (Marcovitch 1923). Since this pioneering study, many examples of photoperiodic response in animals have been described; amongst insects, induction and termination of diapause and the control of seasonal morphs are the most widespread, and have been extensively investigated (Saunders 2002).

Temperature is also a useful cue for timing periodic behaviors that affect reproductive and developmental success. However, temperature can be unreliable, particularly in a terrestrial environment. A survey of daily average temperature and day length in Kyoto, Japan $\left(35.0^{\circ} \mathrm{N}, 135.5^{\circ} \mathrm{E}\right)$ in 2011-2012 and 2012-2013 revealed dramatic day to day fluctuations and interannual variation (Fig. 1). In contrast, photoperiod is perfectly correlated with the time of year without interannual variation. Moreover, compared with temperature, which can undergo abrupt fluctuations, changes in the photoperiod are gradual, which potentially facilitates monitoring of the adaptive responses by organisms. Photoperiodic changes also occur prior to seasonal transitions. The longest day length (i.e., summer solstice) occurs approximately 1.5 month earlier than the period of highest summer temperatures, while the shortest day length (i.e., winter solstice) proceeds the period of lowest winter temperatures by a comparable length of time. As such, organisms can anticipate the seasons much earlier by using photoperiod as a cue.

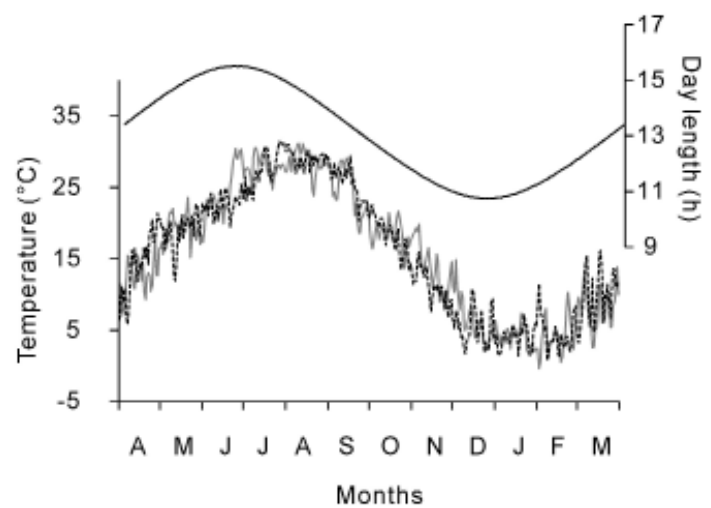

Figure 1. Annual changes in day length (solid line) and temperature during 2011-2012 (grey line) and 2012-2013 (broken line) in Kyoto, Japan (35.0 $\left.\mathrm{N}, 135.5^{\circ} \mathrm{E}\right)$. Day length includes the length of civil twilight. Data are from the Japan Meteorological Agency (2014). There is no interannual difference in day length. 
The significance of photoperiodism in the life history of an organism is illustrated by the example of the bean bug Riptortus pedestris (F.) from Kyoto (Fig. 2). This insect has a photoperiodic reproductive cycle, laying eggs during longer days, while suppressing the development of their reproductive organs and replenishing energy reserves during shorter days (i.e., reproductive diapause). The critical day length (CDL) for the induction of diapause in the Kyoto strain of $R$. pedestris is between 13 and $14 \mathrm{~h}$; that is, insects avert or enter diapause when the day length is longer than $14 \mathrm{~h}$ or shorter than $13 \mathrm{~h}$, respectively (Kobayashi and Numata 1993). The first and second generations of their offspring reproduce during the spring and summer when day lengths are longer than the CDL. However, third generation progeny grow during the autumn, when the day length is shorter than the CDL, enabling adults to enter reproductive diapause and overwinter while deferring oviposition until the following spring (Fig. 2). Thus, the life history of R. pedestris, which is characterized by three generations per year, is regulated by photoperiodism.

Photoperiodic responses have been recorded in many insect species (Danilevskii 1965, Tauber et al. 1986, Danks 1987, Saunders 2002). The physiological mechanisms underlying photoperiodic responses have been discovered by observing responses to natural or unnatural photoperiods, and in some species, the biochemical and molecular components have been identified.

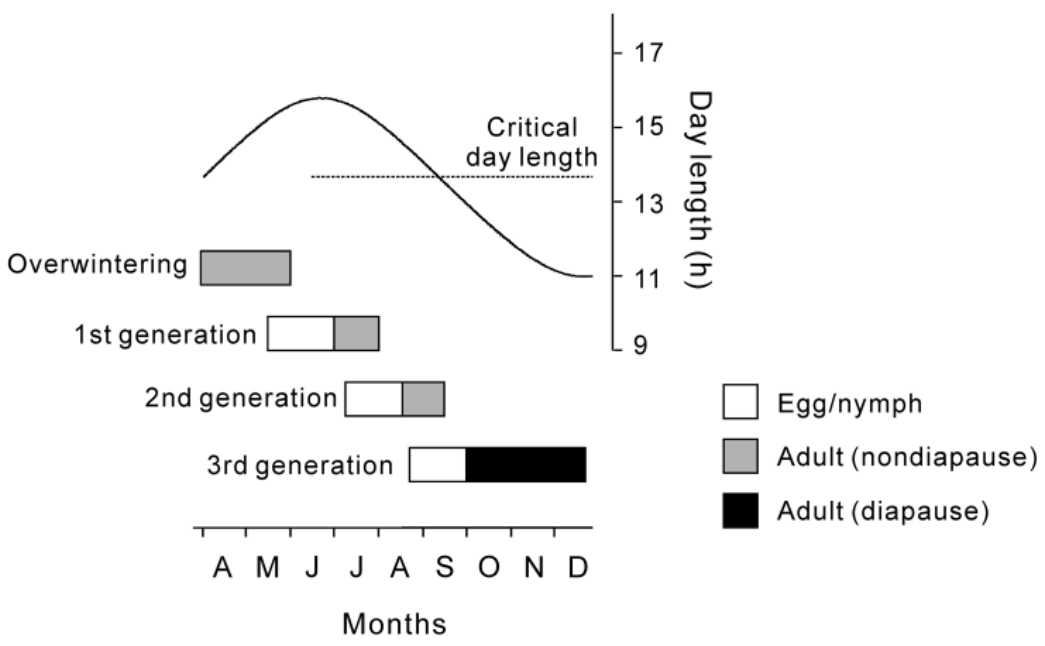

Figure 2. Day length and the life cycle of the bean bug Riptortus pedestris from Kyoto, Japan. Photoperiodism plays a significant role in establishing the life cycle of this species: adults in the first and second generations reproduce in response to long days; however, nymphs and adults in the third generation experience a day length that is shorter than the critical day length, inducing adults to enter diapause and overwinter. 


\section{Geographic Variations in Photoperiodism and Their Genetic Bases}

Geographic variations or clines in photoperiodic responses are present in a large number of insect species (Danilevskii 1965, Saunders 2002). In an exhaustive survey, 41 geographical strains of Drosophila littoralis (Meigen) were collected from localities ranging from the Black Sea coast $\left(41.6^{\circ} \mathrm{N}\right)$ to northern Finland $\left(69.0^{\circ} \mathrm{N}\right)$, revealing geographic variations in CDLs for the induction of adult diapause (Fig. 3, Lankinen 1986). The CDL ranged from 11.6 to $20.3 \mathrm{~h}$ (in the south and north, respectively)and was highly correlated with latitude.

Cross matings between strains from different geographical areas have also been performed to investigate the genetic basis of photoperiodism (Saunders 2002). For example, the St. Petersburg (formerly Leningrad, $\left.59.9^{\circ} \mathrm{N}\right)$ and Sukhumi $\left(43.0^{\circ} \mathrm{N}\right)$ strains of Acronycta rumicis (L.) had CDLs of 19 and $15 \mathrm{~h}$, respectively; their $\mathrm{F}_{1}$ and $\mathrm{F}_{2}$ progeny had an intermediate CDL of approximately $17 \mathrm{~h}$ (Danilevskii 1965). This was similar to the CDL of a population of $A$. rumicis from latitude of $50^{\circ} \mathrm{N}$. These results indicate that a continuous latitudinal gradient exists for CDL-determining gene frequencies with continuous hybridization.

Genes that determine differences in photoperiodism have been identified from studies in the model organism Drosophila melanogaster (Meigen) (Williams et al. 2006, Schmidt et al. 2008). In this species, genetic crosses between geographically distinct strains differing in their potential for diapause led to the isolation of $D p 110$, a gene encoding the insulin signaling pathway component phosphoinositol-3- OH kinase (Williams et al. 2006). The gene couch potato, encoding an RNA-binding protein, was also identified as a major determinant of the diapause phenotype in this species (Schmidt et al. 2008). Although the exact functional mechanisms

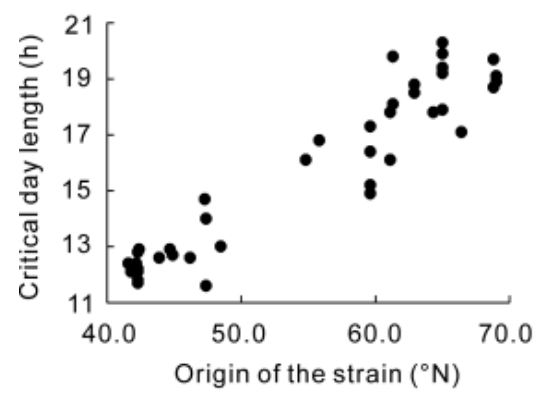

Figure 3. Critical day length (CDL) for the induction of adult diapause in Drosophila littoralis. Each point on the graph represents a strain collected from a different locality ranging from $41.6 \mathrm{~N}$ to $69.0^{\circ} \mathrm{N}$ (data from Lankinen 1986). A high correlation is apparent between CDL and strain origin. 
of these genes are still unknown, they are thought to directly regulate diapause and not photoperiodism. Quantitative trait loci (QTL) analysis is a powerful tool for identifying specific loci or genomic regions in non-model organisms, and in the first QTL map of photoperiodism for the pitcher plant mosquito Wyeomyia smithii (Coquillett), a geographic variation in the photoperiodic control of diapause was revealed: nine QTL for CDL and four for diapause stage were found, although the genes at these loci have yet to be identified (Mathias et al. 2007).

\section{Physiological Cascades and the Molecular Basis of Photoperiodism}

The photoperiodic response in an organism comprises a sequence of several events (Fig. 4, Saunders 2002): (i) photoreception; (ii) assessment of day or night length by a photoperiodic time measurement system;

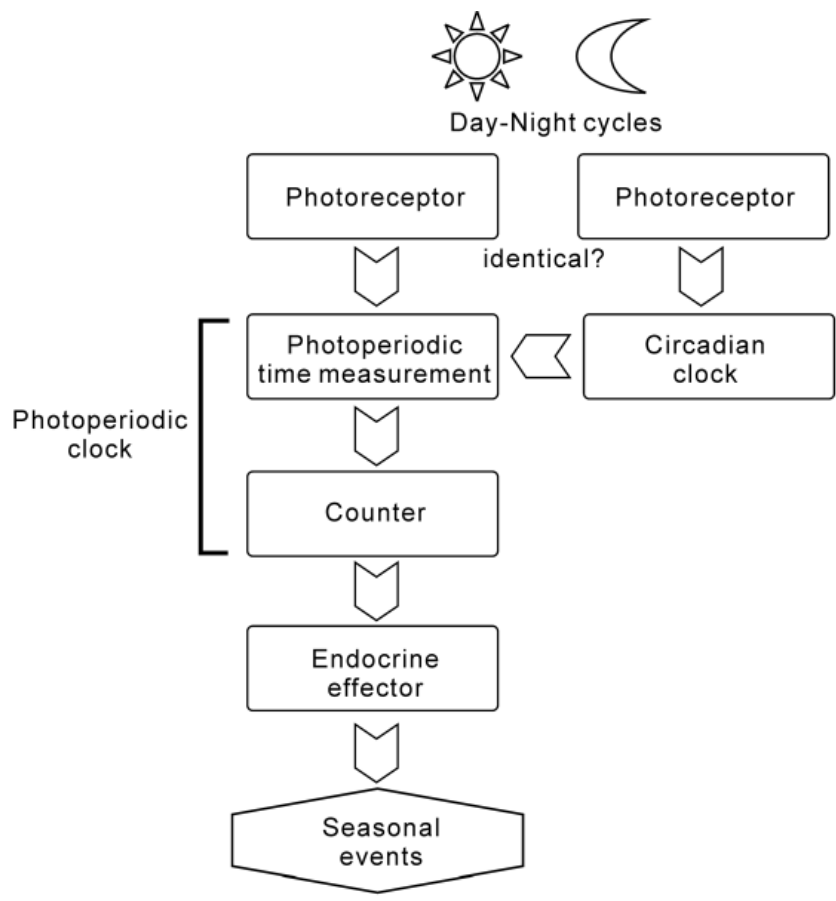

Figure 4. Establishment of photoperiodism through various modules. Light/dark signals are received by photoreceptors for photoperiodism and for the circadian clock, which may or may not be identical depending on the species. The photoperiodic time measurement system measures the length of day or night and involves the circadian clock, while the counter system counts the number of photoperiodic cycles; together, these constitute the photoperiodic clock. When the number of cycles exceeds an internal threshold, the release/ restraint of endocrine effectors is triggered, inducing seasonal events. 


\section{Review Copy - Not for Redistribution Shinsuke Goto - Osaka City University - 10/17/17}

(iii) simultaneous evaluation of the number of photoperiodic cycles by a counter system; and (iv) activation of endocrine effectors that initiate the seasonal event. In this section, each physiological system and the associated molecular mechanism will be discussed in turn.

\section{Photoreceptors}

Visual retinal or nonvisual extraretinal photoreceptors allow organisms to detect photoperiodic information from the environment. Photoperiodic photoreceptors have been described in more than a dozen species from five different orders (Goto et al. 2010). For example, in the blowfly Protophormia terraenovae (Robineau-Desvoidy), photoperiodic induction of adult diapause was lost after the surgical removal of their compound eyes (Shiga and Numata 1997), underscoring the importance of retinal photoreceptors for initiating this physiological process. In contrast, the blowfly Calliphora vicina Robineau-Desvoidy retained photoperiodic sensitivity for the maternal induction of larval diapause after removal of the optic lobes (Saunders and Cymborowski 1996), suggesting that their photoreceptors are extraretinal. Both of these species belong to the family Calliphoridae and demonstrate photoperiodicity as adults, but their distinct photoreceptors suggest that there are no phylogenetic constraints on photoperiodic photoreceptors. It should be noted that retinal and extraretinal photoreception are not mutually exclusive: the stink bug Plautia crossota stali Scott uses both of these for the photoperiodic induction of diapause (Morita and Numata 1999).

Two types of photoreceptive molecules are known in insects: opsin, a class of proteins conjugated to vitamin A-based pigments retinal or 3-hydroxyretinal, and cryptochrome (CRY), a protein conjugated to the vitamin B2-based pigment flavin. Opsins are the major photoreceptive molecules in the retinal photoreceptors. Spectral sensitivity is determined by specific amino acid side chains in the opsin protein, and most species have multiple opsins covering a broad range of wavelengths (Briscoe and Chittka 2001). Carotenoids and their derivatives, including vitamin A, are verified to be essential for photoperiodic responses in various insects and mites, suggesting significance of opsins (Veerman 2001, Saunders 2012). Recent gene knockdown experiments have provided direct support for the role of UV-, blue-, and long-wave-sensitive opsins in the compound eyes of the cricket Modicogryllus siamensis Chopard that are responsible for the photoperiodism of nymphal diapause (Sakamoto and Tomioka 2007, Tamaki et al. 2013). There have been no similar experiments performed in species that use extraretinal photoreceptors; however, in the aphid Megoura viciae (Buckton), in which photoperiodic photoreceptors are restricted to a small area of the protocerebrum (Lees 1964; Steel and Lees 
1977). Gao et al. (1999) detected a crescent-shaped opsin-immunoreactive region in the anterior ventral part of the brain. The opsin-immunoreactive region is not exactly the same as the putative photoreceptor site (Steel and Lees 1977), but they are very close. The long wavelength-sensitive opsin boceropsin is expressed in the cerebral ganglion of Bombyx mori (L.), in which the cerebral ganglion is the photoperiodic photoreceptor, implying that boceropsin is their photoperiodic photoreceptor (Shimizu et al. 2001); however, this observation still awaits functional validation.

CRY absorbs short wavelength light in the range from UV to blue, with little or no sensitivity to wavelengths greater than $500 \mathrm{~nm}$ (Berndt et al. 2007). CRY resets the circadian clock by phase delay or advancement (Stanewsky et al. 1998, see below). In one study, a light pulse delivered during either early or late scotophase prevented pupal diapause in the flesh fly Sarcophaga similis Meade; nonetheless, certain physiological differences were apparent in the responses for each phase, suggesting that distinct mechanisms are involved. The late scotophase was sensitive to a broad range of wavelengths from 395 to $660 \mathrm{~nm}$, indicating that light is perceived by more than one type of opsin. In contrast, the early scotophase detected light at wavelengths of $470 \mathrm{~nm}$ or less, and was insensitive to wavelengths of $583 \mathrm{~nm}$ or greater (Fig. 5); moreover, a greater responsiveness to low light intensity was observed in the early phase. These results imply that different photoreceptors operate in early and late scotophases. Although
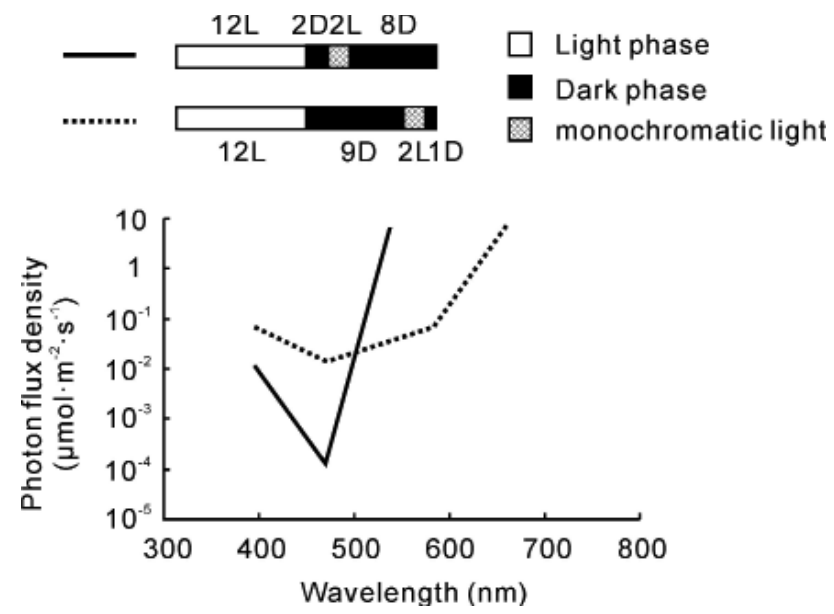

Figure 5. Spectral sensitivity in the flesh fly Sarcophaga similis during night interruption photoperiods with various photon flux densities of monochromatic light. Diapause-destined larvae, which were reared under 12:12 h light/dark (12L:12D) conditions, were exposed to night interruption photoperiods of 12L:2D:2L [monochromatic light]: 8D (solid line) or 12L:9D:2L [monochromatic light]:1D (broken line). Lines in the graph indicate estimated values of photon flux density at which a half of individuals entered diapause [from Goto and Numata (2009b)]. 


\section{Review Copy - Not for Redistribution Shinsuke Goto - Osaka City University - 10/17/17}

the identity of these photoreceptors is unknown, CRY is a candidate photoreceptor for the early scotophase that could act as the photoperiodic time measurement system based on the external coincidence model (see below, Goto and Numata 2009b).

\section{Photoperiodic Time Measurement}

Organisms measure the length of day or night based on information on photoperiod acquired through photoreceptors. Bünning (1936) first proposed the involvement of a circadian clock in photoperiodism. Bünning's hypothesis posited that the 24-h circadian clock consisted of two 12-h half-cycles: the photophil and scotophil (light- and dark-loving phases, respectively) (Fig. 6A), and that short-day effects are observed when light is restricted to the photophil, while long day effects are produced when light penetrates the scotophil. Although this idea is too simple to explain the range of photoperiodic responses in organisms, the basic concept of a circadian clock in photoperiodic time measurement is now widely accepted, not only in insects (Saunders and Bertossa 2011) but also in other organisms from fungi to mammals (Nelson et al. 2010).

Experiments designed to reveal the role of the circadian system in photoperiodic time measurement are based on the known effects of environmental light pulses on the phase shifting and entrainment of circadian oscillations (Saunders 2002). For instance, a short-day light phase of 10-12 h can be coupled with periods of darkness varying from 4 to $72 \mathrm{~h}$ (Nanda and Hamner 1958); alternatively, insects can be exposed to 48- or 72-h cycles consisting of a 12 -h photophase with a light pulse systematically interrupting an extended period of perceived night (Bünsow 1953). In both types of experiment, these aberrant light cycles are repeated throughout the photoperiod-sensitive period, and short-day effects are assessed for each condition at the end of the experiment. A circadian involvement is suspected when short-day effects are observed as occurring in alternating peaks and troughs with 24-h periodicity in the extended night; conversely, the absence of this pattern is evidence for an hour glass-like timer, of which M. viciae (Lees 1973) is an example. However, this can be considered as a heavily dampened circadian oscillator (Saunders 2009), which has been shown to be important for photoperiodic timing even in M. viciae (Vaz Nunes and Hardie 1993). Thus, the functional role of a circadian clock in photoperiodic time measurement is now widely accepted, although some details are still disputed (Bradshaw and Holzapfel 2007b), as discussed below.

A variety of photoperiodic time measurement models have been established by incorporating data accrued under different experimental conditions (Vaz Nunes and Saunders 1999); among these, the external 


\section{A: Bünning's hypothesis}

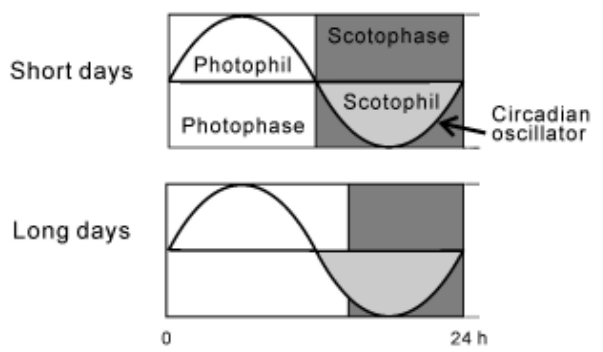

\section{B: External coincidence model}

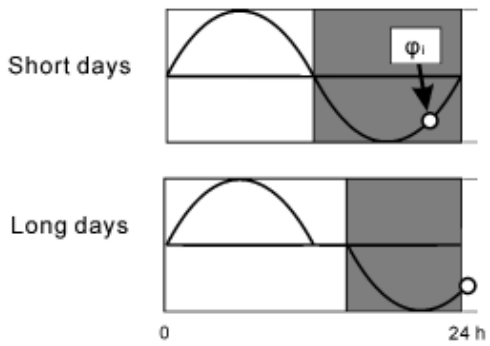

\section{C: Internal coincidence model}
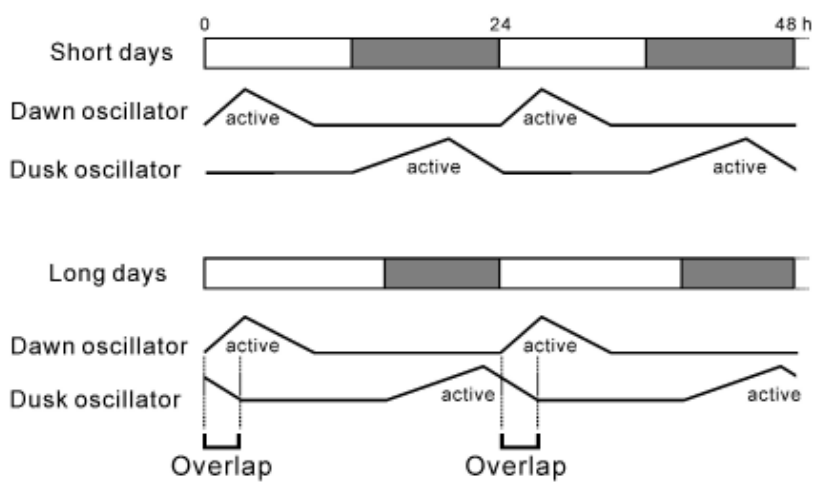

Figure 6. Conceptual diagrams of Bünning's hypothesis and the external and internal coincidence models. (A) In Bünning's hypothesis, the 24-h circadian clock comprises two 12-h half-cycles (photophil and scotophil). Short-day effects are seen when light is restricted to the photophil, while long-day effects are produced when light penetrates the scotophil. (B) Time measurement in the external coincidence model is based on a circadian clock, which sets its phase at dusk and positions the defining light-sensitive phase $(\varphi i)$ in the latter half of scotophase. Under short days, $\varphi \mathrm{i}$ is not exposed to light, eliciting a short-day response; under long days, $\varphi \mathrm{i}$ is exposed to light and a long-day response is induced. (C) The internal coincidence model proposes two oscillators entrained by dawn and dusk, respectively, whose internal phase relationship changes with photophase length. Specific phases for each oscillator are active phases, and long-day responses occur when these overlap. 
and internal coincidence models (Pittendrigh 1960, Pittendrigh and Minis 1964, Tyshchenko 1966) are the most influential. In the former, time measurement is based on a circadian clock that sets its phase at dusk and positions the defining light-sensitive phase ( $\varphi \mathrm{i})$ in the latter half of scotophase (Fig. 6B). During the summer, $\varphi$ i falls in the light period, which represents a longer day to insects and accordingly elicits a longday response. In contrast, during the autumn, $\varphi$ i falls in the dark phase, which is interpreted as a short day and induces a corresponding response (see Saunders 2002, Goto 2013). The internal coincidence model proposes two oscillators that are entrained by dawn and dusk, respectively, with an internal phase that changes with the photophase length (Tyshchenko 1966, Fig. 6C). Certain phases of these oscillators are active phases, and long-day responses are induced when these overlap (see Danilevsky et al. 1970).

\section{Photoperiodic Counter}

Following the measurement of day or night length by the photoperiodic time measurement system, a photoperiodic counter registers successive cycles during the sensitive period until an internal threshold is reached, which triggers a physiological response mediated by endocrine effectors (Saunders 2002). The required day number (RDN) is defined as the number of calendar days or photoperiodic cycles needed to produce a specific seasonal event. The RDN has a capacity for temperature compensation. When females of the parasitic wasp Nasonia vitripennis (Walker) were maintained at various temperatures under a 12:12 h light/dark (12L:12D) cycle, they produced a physiological response associated with short day length and initiated the production of diapause larvae. The uniformity in the response over a range of temperatures suggested that a high degree of temperature compensation had occurred (Saunders 1966). A similar finding was reported for the photoperiodic induction of pupal diapause in the flesh fly (Saunders 1971). In one model of photoperiodic summation, insects accumulate or reduce (under short or long day conditions, respectively) a hypothetical diapause titer in the counter system after processing photoperiodic information in the time measurement system (Gibbs 1975). Thus, a short-day response is elicited upon exceeding the internal threshold, whereas a long day response is induced at subthreshold values (Gibbs 1975). This putative substance is quantitatively accumulated in a photoperiod-dependent manner in some species. For example, S. similis enters pupal diapause in response to short days, but this can be reversed upon long-day exposure: diapause was averted when larvae progressing toward diapause were exposed to $6 \mathrm{~d}$ of 15L:9D or $16 \mathrm{~L}: 8 \mathrm{D}$, indicating that both 15 and $16 \mathrm{~h}$ of light were both interpreted 
as long days. However, after $4 \mathrm{~d}$ of exposure, some larvae failed to avert diapause under 15L:9D conditions, although all averted diapause under 16L:8D (Goto and Numata 2009a). This type of quantitative discrimination of photoperiod can be incorporated into Gibbs's model (Fig. 7, Tagaya et al. 2010).

The molecular components of the photoperiodic counter have been investigated in the cabbage moth Mamestra brassicae (L.) and the giant oak silkmoth Antheraea pernyi (Guérin-Méneville). M. brassicae enters pupal diapause in response to short days, during which dopamine levels are higher in prepupal and early pupal stages. When larvae were fed the dopamine precursor L-dihydroxyphenylalanine during the final instar, diapause was induced even during long days (Noguchi and Hayakawa 1997). These results indicate that dopamine in the hemolymph and nervous system acts as a putative diapause-promoting substance. The

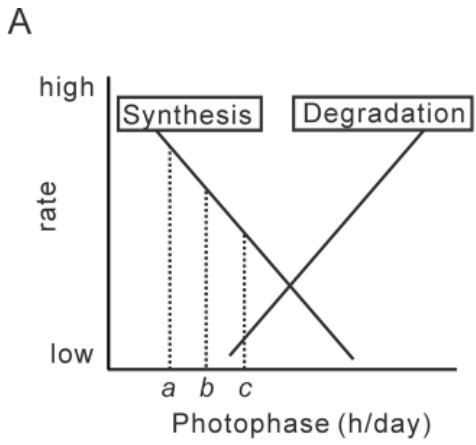

B

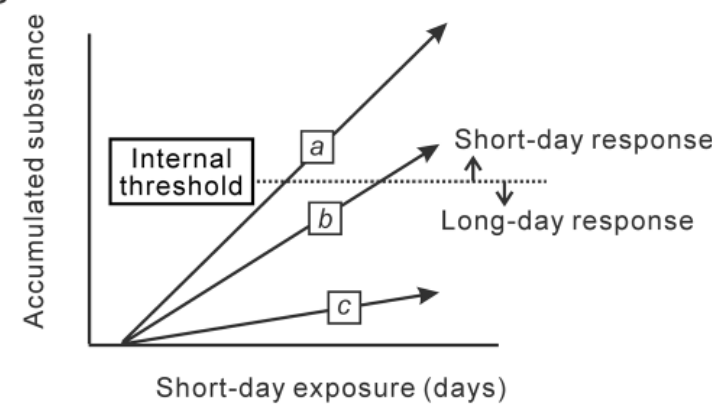

Figure 7. Conceptual diagrams of quantitative photoperiodic time measurement and counter systems. (A) A hypothetical substance is synthesized or degraded according to photoperiod in a quantitative manner (quantitative photoperiodic time measurement). The substance accumulates at a higher rate under shorter (photoperiod a) compared to longer (photoperiod c) days. (B) A short-day response is elicited when the amount of the substance exceeds an internal threshold, but a long-day response is induced for sub-threshold amounts [based on Tagaya et al. (2010)]. 


\section{Review Copy - Not for Redistribution Shinsuke Goto - Osaka City University - 10/17/17}

Receptor for activated protein kinase C1 (Rack 1) gene was upregulated in response to short days as well as dopamine treatment in M. brassicae (Uryu et al. 2002). The response of the Rack 1 gene to short day cycles was quantitative; that is, expression was low for a 1-d and high for a 3-d exposure, while an intermediate response was observed upon exposure to 2 short days. This suggests that Rack 1 is involved in the photoperiodic counter system. Rack 1 protein binds to and stimulates the nuclear translocation of protein kinase $\mathrm{C}$, transducing signals that regulate a variety of cellular functions. In $A$. pernyi, the photoperiodic termination of pupal diapause was induced by injection of melatonin and flupentixol, a dopamine receptor antagonist, even under diapause-promoting short day conditions. Conversely, the injection of dopamine and luzindole, a melatonin receptor antagonist, delayed adult emergence even under diapause-terminating long day conditions. The transition from short to long day conditions was accompanied by the transcriptional upregulation of arylalkylamine $\mathrm{N}$-acetyltransefase (AA-NAT), the rate-limiting enzyme in melatonin synthesis. AA-NAT mRNA levels were also increased by exposure to long days, while the transcript of dopa decarboxylase- - the rate-limiting enzyme for the production of dopamine-decreased. These results suggest that dopamine and melatonin are the key molecules involved in the photoperiodic counter and could potentially function through mutual inhibition (Wang et al. 2014).

\section{Endocrine Effectors}

The hypothetical substance in the photoperiodic counter triggers the release/restraint of endocrine effectors when the internal threshold is exceeded. These effectors have been extensively studied at the molecular level, and the topic has been covered in several recent reviews (e.g., Denlinger et al. 2012, Nylin 2013); therefore, they will not be further addressed here.

\section{Photoperiodic Time Measurement and the Circadian Clock}

It is widely acknowledged that the circadian clock is involved in photoperiodic time measurement (Meuti and Denlinger 2013). Although molecular components of the circadian clock involved in photoperiodism is far from clear, those of the clock governing behavioral rhythmicity have been extensively studied. Such circadian clocks comprise several genes that self-regulate through negative feedback loops (Tomioka and Matsumoto 2010). The core molecular components are well-established, and no alternative, putative clocks have been proposed in insects; thus, it is hypothesized that the molecules of the circadian clock involved in 


\section{Review Copy - Not for Redistribution Shinsuke Goto - Osaka City University - 10/17/17}

photoperiodic time measurement and those regulating circadian behavior are the same. This section provides an overview of the current knowledge of the circadian clock that governs behavioral rhythmicity, before discussing the roles of clock genes in photoperiodism.

\section{Molecular Machinery of the Circadian Clock}

In a highly influential report that paved the way for the molecular dissection of circadian clocks, Konopka and Benzer (1971) reported on three mutants of D. melanogaster with abnormal rhythms of circadianbased adult eclosion and locomotor activity. The mutations were mapped to a single gene known as period (per). Subsequent forward and reverse genetics approaches have led to the discovery of several additional circadian clock genes (Sandrelli et al. 2008, Tomioka and Matsumoto 2010).

The Drosophila circadian clock consists of interlocked transcriptional and translational negative feedback loops (Fig. 8). Positive regulation is provided by the Per-Arnt-Sim domain-containing proteins CLOCK (CLK) and CYCLE (CYC), which heterodimerize and induce the transcription of per, timeless (tim) and other clock-controlled genes. PER and TIM then act as negative regulators by forming a heterodimer that suppresses CYC-CLK activity. This feedback regulation results in an oscillation in the expression levels of the core clock components per and tim. The expression of per and tim mRNA is low during photophase but is elevated during scotophase; protein levels have a similar pattern, but with peaks that are delayed by a few hours compared to those of the transcript. The expression of $\mathrm{Clk}$ mRNA and CLK protein are in antiphase with respect to per and tim mRNA levels; however, there is no oscillation in the expression of $c y c$ transcript or protein.

In insects, two types of CRY are known: a Drosophila-type CRY (CRY-d, also known as CRY1) and a mammalian-type CRY (CRY-m, also known as CRY2). CRY-d is a flavin-based UV- and blue light-sensitive photopigment that induces the degradation of TIM in a light-dependent manner in the central clock. The Drosophila genome does not contain the $c r y-m$ gene, but it has been identified in other insect species. CRY-m does not act as a photoreceptor but as a transcriptional suppressor, possibly working with PER and TIM (Fig. 8) in the monarch butterfly Danaus plexippus (Yuan et al. 2007, Zhu et al. 2008). tim and cry-d are not found in the genomes of the honey bee Apis mellifera or N. vitripennis. Instead, PER is thought to function without TIM in the feedback loop, and opsin-mediated resetting of the clock is expected to play a significant role in these species. Although the diversity of circadian clocks components in insects is recognized, the essential features-positive regulation by CYC and CLK, and negative 

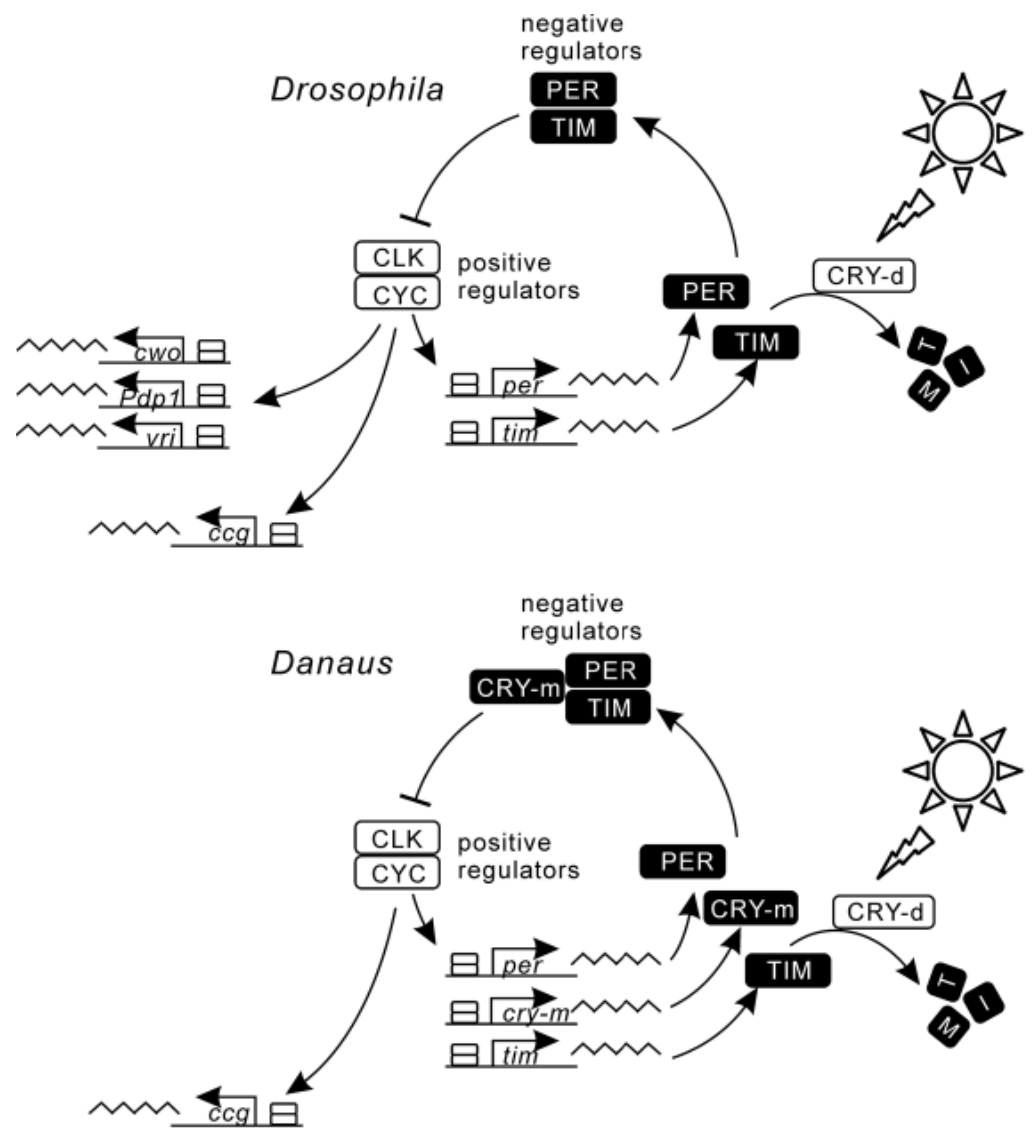

Figure 8. Circadian clock models for Drosophila melanogaster and the monarch butterfly Danaus plexippus. In Drosophila, CLOCK (CLK) and CYCLE (CYC) act as positive regulators to induce transcription of period (per), timeless (tim), and other circadian clock genes participating in the interlocked feedback loops of the clock such as clockwork orange (cwo), Par-domain protein 1 (Pdp1), and vrille (vri), as well as clock-controlled genes (ccg) that mediate downstream signaling cascades. PER and TIM act as negative regulators that inhibit the transcriptional activity of the CYC-CLK heterodimer. Drosophila CRYPTOCHROME (CRY-d) induces the degradation of TIM in a light-dependent manner. Danaus has a nearidentical clock, except that mammalian CRY (CRY-m), which is not found in the Drosophila genome, also acts as a negative regulator by forming a complex with PER and TIM to inhibit the transcriptional activity of CYC-CLK [modified from Yuan et al. (2007), Zhu et al. (2008), and Tomioka et al. (2012)].

feedback provided by PER—are largely conserved among species (Tomioka and Matsumoto 2010). 


\section{Role of Circadian Clock Genes in Photoperiodism}

In an early study of the role of circadian clock genes in photoperiodic time measurement, per null $\left(\mathrm{per}^{0}\right)$ and deletion mutants of D. melanogaster were able to discriminate photoperiods for the induction of diapause (Saunders et al. 1989). This finding suggested that per is not necessary for sensing photoperiods in this species. However, it is premature to conclude that a per-containing circadian clock is dispensable for photoperiodic time measurement, as several lines of evidence suggest otherwise (Koštál 2011); indeed, $p e r^{0}$ mutants had a CDL that was $2 \mathrm{~h}$ shorter than in wild-type (Saunders et al. 1989).

It is now known that tim is involved in the photoperiodic induction of diapause in D. melanogaster. The incidence of diapause in northern European populations of this species is higher than that in the south. Two alleles of tim, s-tim and ls-tim, have been identified, the former producing truncated TIM (S-TIM) and the latter generating both S-TIM and full-length TIM (L-TIM) proteins. Interestingly, the incidence of diapause was altered by introducing natural or artificial tim alleles into the different genetic backgrounds (Tauber et al. 2007): a higher frequency was observed among females carrying $l s$-tim than in those with s-tim for any given photoperiod. In addition, diapause was also induced in the null mutant $t \mathrm{t}^{01}$. There were no significant interactions found between photoperiod and the tim alleles with respect to the incidence of diapause. These results indicate that while tim is not a component of the photoperiodic time measurement system per se, it can confer a predisposition for the regulation of diapause (Bradshaw and Holzapfel 2007b). It is also worth noting that tim $^{01}$ flies exhibit an ambiguous photoperiodic response curve, and enter diapause irrespective of the photoperiod (Tauber et al. 2007). This raises the possibility that tim is directly involved in the photoperiodic time measurement system.

L-TIM was shown to interact with CRY more weakly than S-TIM; as such, flies carrying the ls-tim allele had significantly smaller phase responses in locomotor activity compared to s-tim allele carriers (Sandrelli et al. 2007), which promoted higher levels and amplitude of oscillation of TIM in the ls-tim flies. It is still unclear how the strength of the L-TIM/ CRY interaction contributes to the photoperiodic response or diapause itself. However, it is likely that phase resetting of the clock is involved in seasonal timing.

As a well-established model insect, D. melanogaster provides a variety of genetic tools for the study of photoperiodism; nonetheless, it has several limitations. Diapause in this species is shallow, the incidence varies widely (see Saunders et al. 1989), and it is induced at temperatures that are close to the lower developmental threshold. It was recently determined that in two 


\section{Review Copy - Not for Redistribution Shinsuke Goto - Osaka City University - 10/17/17}

natural populations of $D$. melanogaster representing latitudinal extremes in eastern North America, temperature was the main determinant of ovarian dormancy, while there was no significant effect of photoperiod (Emerson et al. 2009).

An association between clock genes in the photoperiodic induction of diapause has been demonstrated in several other insect species. A genetic variant of the drosophilid fly Chymomyza costata had abnormal photoperiodic response, and failed to enter diapause even under short-day conditions (Riihimaa and Kimura 1988); an arrhythmic pattern of adult eclosion was also observed (Lankinen and Riihimaa 1992), suggesting a causal link between the circadian clock and photoperiodic response. Daily oscillations in per and tim expression were seen in wild-type flies, but per was expressed arrhythmically and at low levels in the variant. In addition, tim mRNA was completely absent (Koštál and Shimada 2001, Pavelka et al. 2003) due to a large deletion in a crucial cis-regulatory element and minimal promoter (Kobelkova et al. 2010). A genetic linkage analysis mapped the gene responsible for the non-diapause phenotype to the locus containing tim (Pavelka et al. 2003), providing evidence for the role of tim in the photoperiodic induction of diapause in C. costata.

RNA interference (RNAi) is a powerful tool that allows the function of a gene of interest to be studied in an organism, and is particularly useful for non-model organisms (Mito et al. 2011) (see also Orchard and Lange this book, Hoffmann et al. this book). This technique was used to examine the role of tim in the photoperiodic response of C. costata; however, due to quite small effect on diapause phenotype, a clear interpretation of the results was not possible (Pavelka et al. 2003). In the cricket M. siamensis, the induction of nymphal diapause is photoperiodic; under short-day conditions, nymphs take more time to reach adults. In this species, per RNAi caused arrhythmic locomotor activity under light-dark conditions and constant darkness, confirming the requirement for per in circadian rhythmicity. In addition, irrespective of the photoperiod, adult emergence patterns upon per knockdown were similar to those of crickets maintained under constant darkness (Sakamoto et al. 2009), indicating that the circadian clock is indispensable for photoperiodic discrimination.

In a series of studies on $R$. pedestris, the expression of per, cry-m, cyc, and Clk was knocked down by RNAi (Ikeno et al. 2010, 2011a,b,c, 2013). Because of the ambiguity of behavioral rhythmicity in this species in the laboratory, cuticle deposition rhythm was used as an output of clock function. The insect endocuticle thickens by the alternating deposition of chitin microfibrils in two different orientations (lamellate and nonlamellate layers) (Fig. 9A), and in R. pedestris and in several other insect species, the rhythm of cuticle deposition is regulated by a circadian clock (Neville 1975, Ito et al. 2008, Ikeno et al. 2010). Disruption of 


\section{A}
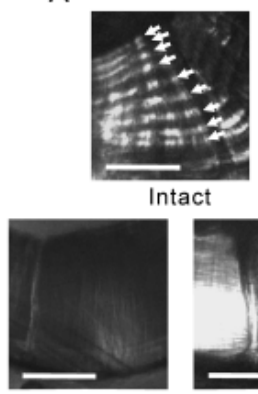

per RNAi

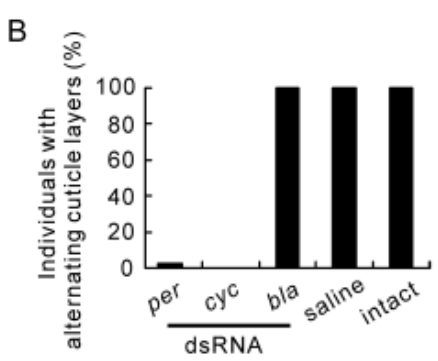

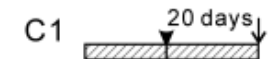
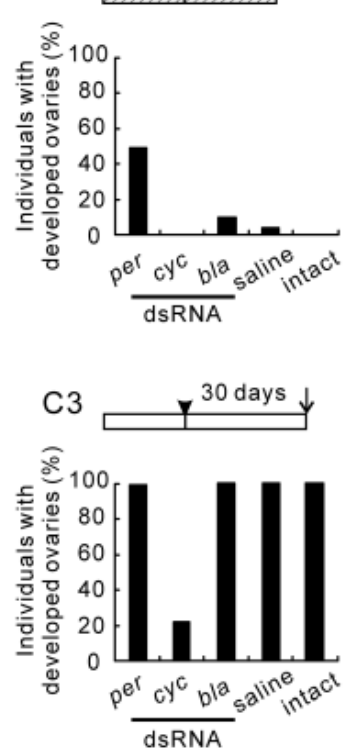
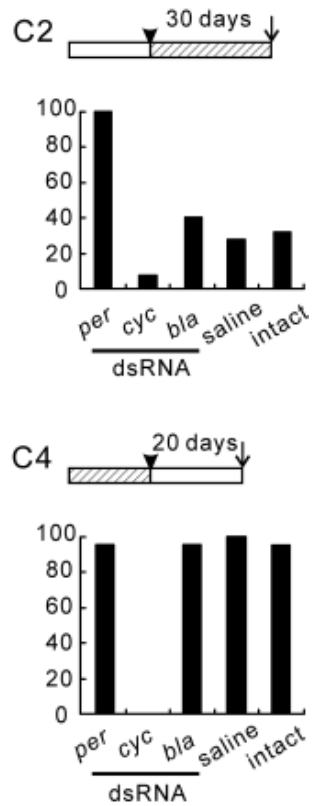

Figure 9. Endocuticle and ovarian development in the bean bug Riptortus pedestris. Gene knockdown via RNA interference (RNAi) was performed by injecting double-stranded RNA (dsRNA) on the day of adult emergence. (A) Cross-sections of the tibia in the hind leg of intact, per RNAi, and cyc RNAi insects $20 \mathrm{~d}$ after emergence. Alternating lamellate (arrows) and non-lamellate layers of the endocuticle are observed in intact insects. Knockdown of per or cyc produced a single, thickened non-lamellate or lamellate layer, respectively. Scale bar, $25 \mu \mathrm{m}$. (B) Fraction of insects with normal, alternating cuticle layers $20 \mathrm{~d}$ after the injection of per, cyc, or $\beta$-lactamase (bla; negative control) dsRNA or saline. (C) Effects of per and cyc RNAi on ovarian development. The experimental schedules are shown as horizontal hatched and open bars (short- and long-day conditions, respectively). Arrowheads indicate the day of adult emergence and of dsRNA injection, and arrows indicate the day of dissection. Insects were maintained under short-day conditions (C1), transferred from long- to short-day conditions (C2), maintained under long-day conditions (C3), or transferred from short- to long-day conditions (C4) [from Ikeno et al. (2010)].

either negative or positive regulators arrests the clock and results in the production of single layers with distinct phenotypes: when per or $c r y-m$, negative regulators, are knocked down, only a thick non-lamellate layer is produced, whereas the silencing of $c y c$ and $C l k$, positive regulators, gives rise to only a lamellate layer (Fig. 9A,B). RNAi-mediated knockdown of the negative regulators failed to suppress CYC-CLK activity, causing consistent activation of the downstream cascade; this was in turn suppressed by inhibiting the positive regulators, as evidenced by the expression of other clock genes (Ikeno et al. 2010, 2011a,b). Thus, the removal of either the positive or negative regulators caused the clock to 
stop its oscillation, but stuck the clock at distinct phases, causing a specific phenotypic output.

In females of $R$. pedestris, per and cry- $m$ RNAi promoted ovarian development even under diapause-inducing short-day conditions, which was suppressed by $c y c$ and Clk RNAi even under diapause-averting long day conditions (Ikeno et al. 2010, 2011b, 2013, Fig. 9C). The different phenotypes induced by silencing negative or positive regulators indicate that the arrest of the circadian clock at specific phases activates distinct signaling cascades that govern photoperiodic response, providing evidence that circadian clock genes are involved in photoperiodic time measurement.

\section{Bünning's Hypothesis and Alternative Hypothesis}

Given the participation of the circadian clock in photoperiodism, the question that arises is which processes in the photoperiodic response are affected. On the basis of Bünning's hypothesis, the circadian clock is actively involved in photoperiodic time measurement; however, it is possible that other processes in the photoperiodic response are also under circadian control independent of this clock, and directly induces seasonal events. Support for this idea comes from the observation that the circadian clock regulates a wide array of physiological processes in an organism, such as behavior, learning, feeding, metabolism, chemosensation, and immunity (Allada and Chung 2010). It is also known that in addition to a central circadian oscillator in the brain, peripheral clocks exist in a variety of organs, including the compound eyes, antennae, wings, legs, Malpighian tubules, and epidermis (Ito et al. 2008). These oscillators have an inherent rhythmicity that is independent of the central clock (Tomioka et al. 2012). In experiments where RNAi is introduced into an organism by feeding or injection, gene knockdown is not tissue- or cell type-specific, and it is therefore not possible to identify whether the central or peripheral clocks are being affected (Bradshaw and Holzapfel 2010). Recent studies examining the function of clock genes in peripheral tissues in relation to photoperiodism found that there they played significant "non-circadian" role (Bajgar et al. 2013a,b).

Like R. pedestris, the linden bug Pyrrhocoris apterus belongs to the infraorder Pentatomomorpha and enters reproductive diapause in response to short days. RNAi of per induced oviposition in about onethird of females maintained under conditions promoting diapause, but silencing $c y c$ or $c r y-m$ had no effect on reproduction (Bajgar et al. 2013a). cry-m expression was highly upregulated in the gut of P. apterus in diapause, while that of the circadian clock gene Par domain protein 1 (Pdp1) was concomitantly reduced. The juvenile hormone $(\mathrm{JH})$ receptor 


\section{Review Copy - Not for Redistribution Shinsuke Goto - Osaka City University - 10/17/17}

Methoprene-tolerant (Met), $\mathrm{Clk}$ and $c y c$ are all required in the gut to activate the Pdp1 gene during reproduction and to simultaneously suppress $c r y-m$ gene that promotes the diapause program (Bajgar et al. 2013b). Reproductive diapause is induced in a wide variety of insect species in the absence of JH. In P. apterus maintained under long-day conditions, ablation of the corpus allatum-the site of JH synthesis and secretioninduced diapause-specific expression patterns of the circadian clock genes in the gut (Bajgar et al. 2013a), implying that their expression is regulated by JH. Thus, while CYC, CLK, PDP1, and CRY-m play no role in regulating the peripheral circadian clock in the gut, PDP1 and CRY-m are nonetheless under the control of a hormonal signal that dictates the diapause- or nondiapause-specific physiological state of the organ and CYC and CLK works with MET to regulate the JH signaling (Bajgar et al. 2013b).

Although these results emphasize the role of circadian clock genes in the peripheral tissues as the output system of photoperiodism, they do not exclude their involvement in a photoperiodic time measurement system within the brain, which is discussed in the following section.

\section{Location of the Circadian Clock for Photoperiodism}

Microcautery experiments have determined that neurosecretory cells (NSCs) located at the anterior extremity of the protocerebrum (Group I NSCs) secrete effectors that control photoperiodic regulation of virginopara production in M. viciae (Steel and Lees 1977). Lesioning a region lateral to Group I NSCs also effectively disrupted the normal photoperiodic response. On the basis of these observations, this region has been assumed to harbor the photoperiodic clock; however, clear functional evidence is still lacking.

Immunocytochemical approaches were used to map neurons expressing PER, TIM, and the neuropeptide prothoracicotropic hormone (PTTH) in A. pernyi, which enters pupal diapause in response to short days (Sauman and Reppert 1996a,b). PTTH triggers the synthesis and secretion of ecdysteroids, and in most cases the arrest of PTTH secretion is the critical determinant of larval and pupal diapause (Denlinger et al. 2012). In A. pernyi, PER and TIM were coexpressed in the cytoplasm and axons of eight cells in the dorsal lateral protocerebrum of pupae and adults. A pair of PER-positive cells was located adjacent to the PTTH-expressing NSCs; their physical proximity and the extensive dendritic arborization of the NSCs in this region suggest routes of communication between these two cell populations that are important for the circadian control of PTTH release (Sauman and Reppert 1996a,b). However, there is no report on a link between these cells and photoperiodic responses in this species. In the tobacco hornworm Manduca sexta (L.), four NSCs in the pars lateralis 


\section{Review Copy - Not for Redistribution Shinsuke Goto - Osaka City University - 10/17/17}

(PL) of each brain hemisphere are immunoreactive for PER. These cells were identified as type-Ia1 NSCs that produce the neuropeptide hormone corazonin (Wise et al. 2002). When these cells were removed under diapause-inducing short-day conditions, the incidence of nondiapause pupae increased relative to controls (Shiga et al. 2003). The dendrites of type-Ia1 and PTTH cells coexist in the same region of neuropil (Shiga et al. 2003), and intracellular staining of lateral NSCs has revealed that their dendritic fields overlap (Carrow et al. 1984). This provides a basis for possible paracrine or synaptic inhibition of the PTTH NSCs by type-Ia1 cells, which would implicate the latter as an element of the photoperiodic time measurement system.

In P. terraenovae, neurons with cell bodies in the PL projecting to the retrocerebral complex (designated as PL neurons) are necessary for the induction of reproductive diapause under short-day and low temperature conditions (Shiga and Numata 2000). Synaptic connections exist between PL neurons and pigment-dispersing factor (PDF)-immunoreactive neurons (Hamanaka et al. 2005). PDF is considered to function as an output signal from the circadian clock to regulate locomotor activity (Renn et al. 1999). Significant role of PDF in the circadian rhythm has also been reported in other insect species (Petri and Stengl 1997, Lee et al. 2009, Hassaneen et al. 2011). In P. terraenovae, PDF-immunoreactive fibers with synaptic connections to PL neurons probably originate from PDF-expressing neurons whose cell bodies are located at the base of the medulla in the optic lobe (Hamanaka et al. 2005). These neurons, called s-LNvs, were also immunoreactive for PER; when the brain region containing s-LNvs was removed, flies showed arrhythmic activity patterns under constant darkness, and failed to discriminate photoperiods, suggesting that s-LNvs, which function as circadian clock neurons in the brain, are also responsible for photoperiodic time measurement (Shiga and Numata 2009).

A similar neural circuit governing photoperiodism also exists in R. pedestris (Fig. 10, Ikeno et al. 2014). PDF-immunoreactive neurons in this insect have cell bodies located in the optic lobe, with fibers extending to the protocerebrum. Surgical removal of the region containing PDF-positive cell bodies disrupted the photoperiodic regulation of diapause (Fig. 10), although RNAi-mediated silencing of $p d f$ expression had no effect on photoperiodic response. This suggests that PDF-expressing neurons, which are involved in circadian regulation, mediate photoperiodic responses in R. pedestris through a factor other than PDF. Although the identification of these neurons as definitive circadian clock components in this species awaits confirmation, their morphological and biochemical similarities to PDF-secreting neurons in other insects provide strong support for this possibility (Stengl and Homberg 1994, Vafopoulou et al. 2010, Vafopoulou and Steel 2012, see also the references in Ikeno et al. 2014). 


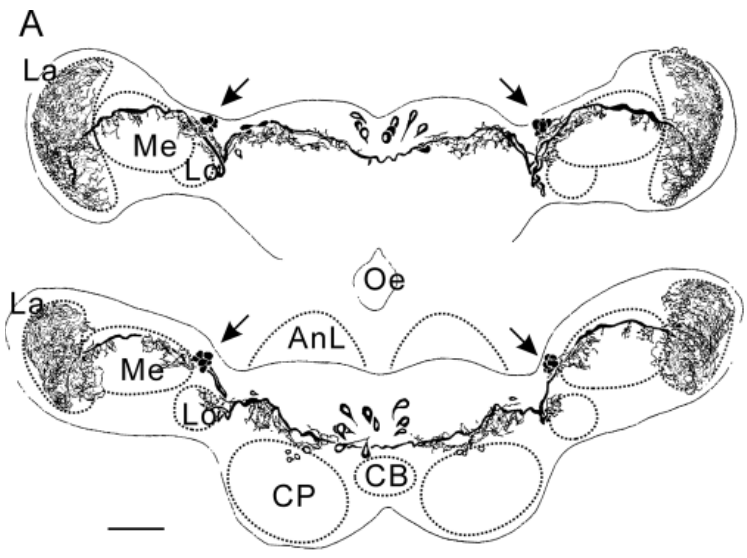

B
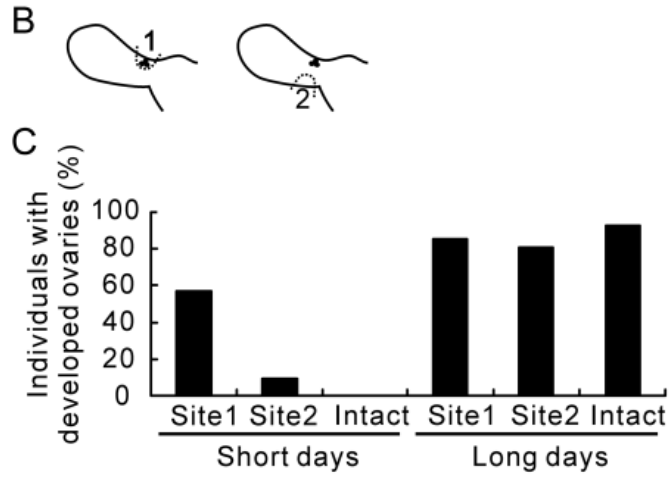

Figure 10. Pigment-dispersing factor (PDF)-immunoreactive neurons in the brain of the bean bug Riptortus pedestris and effect of their removal on photoperiodism. (A) Tracings of PDFexpressing neurons in the brain (top, anterior view; bottom, dorsal view). Arrows indicate cell bodies. AnL, antennal lobe; CB, central body; CP, corpus pedunculatus; La, lamina; Lo, lobulla; Me, medulla; Oe, oesophagus. Scale bar, $100 \mu \mathrm{m}$. (B) Schematic illustrations of ablated optic lobe regions. Anterior and posterior bases of the medulla are designated as Sites 1 and 2, respectively. PDF-positive neurons are shown as closed circles. (C) Effect of eliminating Sites 1 or 2 on photoperiodic induction of ovarian development. The brain regions were surgically removed in insects reared under diapause-inducing short-day conditions, after which they were maintained under the same conditions or transferred to diapauseaverting long-day conditions [from Ikeno et al. (2014)].

\section{Future Prospects}

Endocrine effectors that direct seasonal events in an organism have been extensively studied at the molecular level (Denlinger et al. 2012). Recently, evidence for the causal involvement of opsins in photoperiodic photoreception was obtained (Tamaki et al. 2013), while the role of CRY 


\section{Review Copy - Not for Redistribution Shinsuke Goto - Osaka City University - 10/17/17}

is still uncertain. In contrast, the molecular mechanisms underlying photoperiodic time measurement and counter systems are still obscure.

A remaining point of contention in the photoperiodic time measurement system is whether components of the circadian clock are involved. The principles outlined by Bünning's hypothesis are now widely accepted for various organisms (Nelson et al. 2012), and to date, no alternatives to a model involving per, $\mathrm{Clk}$, and cyc have been proposed in the circadian clock. Because circadian rhythmicity and photoperiodism are both dependent on photoperiod, linking the mechanisms that govern daily and seasonal timing has intrinsic appeal. Indeed, data from several insect species support a functional connection, although others have cautioned against drawing too many parallels because of the existence of some discrepancies (Bradshaw and Holzapfel 2012). For example, one study examined the genetic linkage between the circadian clock and photoperiodic time measurement system in D. littoralis (Lankinen and Forsman 2006). A northern strain with a long CDL for diapause, early phase of entrained eclosion rhythm in response to extremely short days, and a short period for the free-running eclosion rhythm was crossed with a southern strain with a short CDL, late eclosion phase, and long free-running period. After many generations, during which free recombination, artificial selection, and genetic drift occurred, a novel strain with diapause and eclosion rhythm characteristic of the southern and northern strains, respectively, was produced. The complete separation of the circadian-based eclosion rhythm from photoperiodic behavior revealed that independent mechanisms underlie these two processes. A similar incongruence between circadian rhythmicity and photoperiodism was reported in W. smithii (see Bradshaw and Holzapfel 2007a, 2012 and references herein). However, an alternative explanation is that a single oscillator is responsible for both processes, and that circadian or photoperiodic responses are affected through distinct downstream pathways (Lankinen and Forsman 2006, Goto 2013).

On the basis of the existing data, the most parsimonious approach to elucidating the photoperiodic time measurement system is to assume the involvement of the circadian clock genes, particularly as there is still no clear evidence to the contrary. It is also useful to approach the problem using forward genetics in order to identify non-circadian genes that are involved in photoperiodic time measurement. As photoperiodic responses have evolved multiple times in insects and the core circadian clock differs among insect taxa, it is likely that circadian clocks play species-specific roles in photoperiodic responses. Comparative studies involving diverse insect species are therefore welcome.

Apart from the question of whether circadian clock genes participate in photoperiodic time measurement, there is still a lack of basic understanding about process of photoperiodic time measurement itself. For instance, 


\section{Review Copy - Not for Redistribution \\ Shinsuke Goto - Osaka City University - 10/17/17}

the physiological entity represented by $\varphi \mathrm{i}$ in the external coincidence model has yet to be identified; in the internal coincidence model, the components that measure the overlap between the two oscillators are still unclear. Similarly, in the counter system, the accumulation of hypothetical substances and existence of internal thresholds have been hypothesized, but these concepts still require substantiation with physiological evidence. Dopamine, melatonin, and the Rack 1 gene have all been proposed to be involved (Noguchi and Hayakawa 1997, Uryu et al. 2003, Wang et al. 2014) but functional experiments are required in order to confirm their roles.

In conclusion, there is still much to be discerned in the cascade of photoperiodism, and a molecular dissection of this process represents a promising direction in the field. The integration of experimental approaches such as classic physiological experiments, fine-scale mapping of genes by QTL analyses, genome-editing and high-throughput sequencing technologies, which would be particularly powerful for non-model organisms, and loss of function experiments using RNAi will greatly enhance future studies on photoperiodism.

\section{Acknowledgements}

We thank Dr. Sakiko Shiga at Osaka City University for critical reading of this manuscript. This work was supported in part by Grants-in-Aid for Scientific Research from the Japan Society for the Promotion of Science to H.N. (20380038) and to S.G.G. (25450488).

Keywords: Photoperiodism, photoreceptor, photoperiodic time measurement, circadian clock, counter, endocrine effectors, diapause, Bünning's hypothesis, brain, day length, circadian clock genes, opsin, cryptochrome, pigment-dispersing factor

\section{References}

Allada, R. and B.Y. Chung. 2010. Circadian organization of behavior and physiology in Drosophila. Annu. Rev. Physiol. 72: 605-624.

Bajgar, A., D. Dolezel and M. Hodkova. 2013a. Endocrine regulation of non-circadian behavior of circadian genes in insect gut. J. Insect Physiol. 59: 881-886.

Bajgar, A., M. Jindra and D. Dolezel. 2013b. Autonomous regulation of the insect gut by circadian genes acting downstream of juvenile hormone signaling. Proc. Natl. Acad. Sci. USA 110: 4416-4421.

Berndt, A., T. Kottke, H. Breitkreuz, R. Dvorsky, S. Hennig, M. Alexander and E. Wolf. 2007. A novel photoreaction mechanism for the circadian blue light photoreceptor Drosophila cryptochrome. J. Biol. Chem. 282: 13011-13021.

Bradshaw, W.E. and C.M. Holzapfel. 2007a. Evolution of animal photoperiodism. Annu. Rev. Ecol. Evol. Sys. 38: 1-25.

Bradshaw, W.E. and C.M. Holzapfel. 2007b. Tantalizing timeless. Science 316: 1851-1852. 
Bradshaw, W.E. and C.M. Holzapfel. 2010. Circadian clock genes, ovarian development and diapause. BMC Biol. 8: 115.

Bradshaw, W.E., K.J. Emerson and C.M. Holzapfel. 2012. Genetic correlations and the evolution of photoperiodic time measurement within a local population of the pitcherplant mosquito, Wyeomyia smithii. Heredity 108: 473-479.

Briscoe, A.D. and L. Chittka. 2001. The evolution of color vision in insects. Annu. Rev. Entomol. 46: 471-510.

Bünning, E. 1936. Die endogene Tagesrhythmik als Grundlage der photoperiodischen Reaktion. Ber. Dtsch. Bot. Ges. 54: 590-607.

Bünsow, R.C. 1953. Uber tages- und jahresrhythmische Änderungen der photoperiodischen Lichtempfindlichkeit bei Kalanchoe blossfeldiana und ihre Beziehungen zur endogonen Tagesrhythmik. Z. Bot. 41: 257-276.

Carrow, G.M., R.L. Calabrese and C.M. Williams. 1984. Architecture and physiology of insect cerebral neurosecretory cells. J. Neurosci. 4: 1034-1044.

Danilevskii, A.S. 1965. Photoperiodism and Seasonal Development of Insects. Oliver \& Boyd, Edinburgh.

Danilevsky, A.S., N.I. Goryshin and V.P. Tyshchenko. 1970. Biological rhythms in terrestrial arthropods. Annu. Rev. Entomol. 15: 201-244.

Danks, H.V. 1987. Insect Dormancy: An Ecological Perspective. Biological Survey of Canada, Ottawa.

Denlinger, D.L., G.D. Yocum and J.P. Rinehart. 2012. Hormonal control of diapause. pp. 430-463. In: L.I. Gilbert (ed.). Insect Endocrinology. Academic Press, London.

Emerson, K.J., A.M. Uyemura, K.L. McDaniel, P.S. Schmidt, W.E. Bradshaw and C.M. Holzapfel. 2009. Environmental control of ovarian dormancy in natural populations of Drosophila melanogaster. J. Comp. Physiol. A 195: 825-829.

Gao, N., M. von Schantz, R.G. Foster and J. Hardie. 1999. The putative brain photoperiodic photoreceptors in the vetch aphid, Megoura viciae. J. Insect Physiol. 45: 1011-1019.

Garner, W.W. and H.A. Allard. 1920. Effect of the relative length of day and night and other factors of the environment on growth and reproduction in plants. J. Agric. Res. 18: 553-606.

Gibbs, D. 1975. Reversal of pupal diapause in Sarcophaga argyrostoma by temperature shifts after puparium formation. J. Insect Physiol. 21: 1179-1186.

Goto, S.G. 2013. Roles of circadian clock genes in insect photoperiodism. Entomol. Sci. 16: $1-16$.

Goto, S.G., S. Shiga and H. Numata. 2010. Perception of light and the role of clock genes. pp. 258-286. In: R.J. Nelson, D.L. Denlinger and D.E. Somers (eds.). Photoperiodism: The Biological Calendar. Oxford University Press, Oxford.

Goto, S.G. and H. Numata. 2009a. Alteration of the pupal diapause program and regulation of larval duration by photoperiod in the flesh fly Sarcophaga similis Meade (Diptera: Sarcophagidae). Appl. Entomol. Zool. 44: 603-609.

Goto, S.G. and H. Numata. 2009b. Possible involvement of distinct photoreceptors in the photoperiodic induction of diapause in the flesh fly Sarcophaga similis. J. Insect Physiol. 55: 401-407.

Hamanaka, Y., K. Yasuyama, H. Numata and S. Shiga. 2005. Synaptic connections between pigment-dispersing factor-immunoreactive neurons and neurons in the pars lateralis of the blow fly, Protophormia terraenovae. J. Comp. Neurol. 491: 390-399.

Hassaneen, E., A. El-Din Sallam, A. Abo-Ghalia, Y. Moriyama, S.G. Karpova, S. Abdelsalam, A. Matsushima, Y. Shimohigashi and K. Tomioka 2011. Pigment-dispersing factor affects nocturnal activity rhythms, photic entrainment, and the free-running period of the circadian clock in the cricket Gryllus bimaculatus. J. Biol. Rhythms 26: 3-13.

Ikeno, T., H. Numata and S.G. Goto. 2008. Molecular characterization of the circadian clock genes in the bean bug, Riptortus pedestris, and their expression patterns under long- and short-day conditions. Gene 419: 56-61. 
Ikeno, T., S.I. Tanaka, H. Numata and S.G. Goto. 2010. Photoperiodic diapause under the control of circadian clock genes in an insect. BMC Biol. 8: 116.

Ikeno, T., C. Katagiri, H. Numata and S.G. Goto. 2011a. Causal involvement of mammaliantype cryptochrome in the circadian cuticle deposition rhythm in the bean bug Riptortus pedestris. Insect Mol. Biol. 20: 409-415.

Ikeno, T., H. Numata and S.G. Goto. 2011b. Photoperiodic response requires mammalian-type cryptochrome in the bean bug Riptortus pedestris. Biochem. Biophys. Res. Commun. 410: 394-397.

Ikeno, T., H. Numata and S.G. Goto. 2011c. Circadian clock genes period and cycle regulate photoperiodic diapause in the bean bug Riptortus pedestris males. J. Insect Physiol. 57: 935-938.

Ikeno, T., H. Numata, S.G. Goto and S. Shiga. 2014. The involvement of the brain region containing pigment-dispersing factor-immunoreactive neurons in the photoperiodic response of the bean bug Riptortus pedestris. J. Exp. Biol. 217: 453-462.

Ito, C., S.G. Goto, S. Shiga, K. Tomioka and H. Numata. 2008. Peripheral circadian clock for the cuticle deposition rhythm in Drosophila melanogaster. Proc. Natl. Acad. Sci. USA 105: 8446-8451.

Japan Meteorological Agency. 2014. URL http://www.jma.go.jp/jma/indexe.html.

Kobayashi, S. and H. Numata. 1993. Photoperiodic responses controlling the induction of adult diapause and the determination of seasonal form in the bean bug, Riptortus clavatus. Zool. Sci. 10: 983-990.

Konopka, R.J. and S. Benzer. 1971. Clock mutants of Drosophila melanogaster. Proc. Natl. Acad. Sci. USA 68: 2112-2116.

Koštál, V. 2011. Insect photoperiodic calendar and circadian clock: Independence, cooperation, or unity? J. Insect Physiol. 57: 538-556.

Koštál, V. and K. Shimada. 2001. Malfunction of circadian clock in the non-photoperiodicdiapause mutants of the drosophilid fly, Chymomyza costata. J. Insect Physiol. 11: $1269-1274$.

Kobelková, A., A. Bajgar and D. Dolezel. 2010. Functional molecular analysis of a circadian clock gene timeless promoter from the drosophilid fly, Chymomyza costata. J. Biol. Rhythms 25: 399-409.

Lankinen, P. 1986. Geographical variation in circadian eclosion rhythm and photoperiodic adult diapause in Drosophila littoralis. J. Comp. Physiol. A 159: 123-142.

Lankinen, P. and P. Forsman. 2006. Independence of genetic geographical variation between photoperiodic diapause, circadian eclosion rhythm and Thr-Gly repeat region of the period gene in Drosophila littoralis. J. Biol. Rhythms 21: 3-12.

Lankinen, P. and A.J. Riihimaa. 1992. Weak circadian eclosion rhythmicity in Chymomyza costata (Diptera: Drosophilidae), and its independence of diapause type. J. Insect Physiol. 38: 803-811.

Lee, C.M., M.T. Su and H.J. Lee. 2009. Pigment dispersing factor: an output regulator of the circadian clock in the German cockroach. J. Biol. Rhythms 24: 35-43.

Lees, A.D. 1964. The location of the photoperiodic receptors in the aphid Megoura viciae Buckton. J. Exp. Biol. 41: 119-133.

Lees, A.D. 1973. Photoperiodic time measurement in the aphid Megoura viciae. J. Insect Physiol. 19: 2279-2316.

Marcovitch, S. 1923. Plant lice and light exposure. Science 58: 537-538.

Mathias, D., L. Jacky, W.E. Bradshaw and C.M. Holzapfel. 2007. Quantitative trait loci associated with photoperiodic response and stage of diapause in the pitcher-plant mosquito, Wyeomyia smithii. Genetics 176: 391-402.

Meuti, M.E. and D.L. Denlinger. 2013. Evolutionary links between circadian clocks and photoperiodic diapause in insects. Integr. Comp. Biol. 53: 131-143.

Mito, T., T. Nakamura, T. Bando, H. Ohuchi and S. Noji. 2011. The advent of RNA interference in Entomology. Entomol. Sci. 14: 1-8. 


\section{Insect Molecular Biology and Ecology}

Morita, A. and H. Numata. 1999. Localization of the photoreceptor for photoperiodism in the stink bug, Plautia crossota stali. Physiol. Entomol. 24: 189-195.

Nanda, K.K. and K.C. Hamner. 1958. Studies on the nature of the endogenous rhythm affecting photoperiodic response of Biloxi soybean. Bot. Gaz. 120: 14-25.

Nelson, R.J., D.L. Denlinger and D.E. Somers (eds.). 2010. Photoperiodism: Biological Calendar. Oxford University Press, New York.

Neville, A.C. 1975. Biology of the Arthropod Cuticle. Springer, Berlin.

Noguchi, H. and Y. Hayakawa. 1997. Role of dopamine at the onset of pupal diapause in the cabbage armyworm Mamestra brassicae. FEBS Lett. 413: 157-161.

Nylin, S. 2013. Induction of diapause and seasonal morphs in butterflies and other insects: knowns, unknowns and the challenge of integration. Physiol. Entomol. 38: 96-104.

Pavelka, J., K. Shimada and V. Koštál. 2003. TIMELESS: a link between fly's circadian and photoperiodic clocks? Eur. J. Entomol. 100: 255-265.

Petri, B. and M. Stengl. 1997. Pigment-dispersing hormone shifts the phase of the circadian pacemaker of the cockroach Leucophaea maderae. J. Neurosci. 17: 4087-4093.

Pittendrigh, C.S. 1960. Circadian rhythms and the circadian organization of living systems. Cold Spring Harb. Symp. Quant. Biol. 25: 159-184.

Pittendrigh, C.S. and D.H. Minis. 1964. The entrainment of circadian oscillations by light and their role as photoperiodic clocks. Am. Nat. 98: 261-294.

Renn, S.C., J.H. Park, M. Rosbash, J.C. Hall and P.H. Taghert. 1999. A pdf neuropeptide gene mutation and ablation of PDF neurons each cause severe abnormalities of behavioral circadian rhythms in Drosophila. Cell 99: 791-802.

Riihimaa, A.J. and M.T. Kimura. 1988. A mutant strain of Chymomyza costata (Diptera: Drosophilidae) insensitive to diapause-inducing action of photoperiod. Physiol. Entomol. 13: 441-445.

Sakamoto, T. and K. Tomioka. 2007. Effects of unilateral compound-eye removal on the photoperiodic responses of nymphal development in the cricket Modicogryllus siamensis. Zool. Sci. 24: 604-610.

Sakamoto, T., O. Uryu and K. Tomioka. 2009. The clock gene period plays an essential role in photoperiodic control of nymphal development in the cricket Modicogryllus siamensis. J. Biol. Rhythms 24: 379-390.

Sandrelli, F., R. Costa, C.P. Kyriacou and E. Rosato. 2008. Comparative analysis of circadian clock genes in insects. Insect Mol. Biol. 17: 447-463.

Sandrelli, F., E. Tauber, M. Pegoraro, G. Mazzotta, P. Cisotto, J. Landskron, R. Stanewsky, A. Piccin, E. Rosato, M. Zordan, R. Costa and C.P. Kyriacou. 2007. A molecular basis for natural selection at the timeless locus in Drosophila melanogaster. Science 316: 1898-1900.

Sauman, I. and S.M. Reppert. 1996a. Circadian clock neurons in the silkmoth Antheraea pernyi: novel mechanisms of period protein regulation. Neuron 17: 889-900.

Sauman, I. and S.M. Reppert. 1996b. Molecular characterization of prothoracicotropic hormone (PTTH) from the giant silkmoth Antheraea pernyi: Developmental appearance of PTTH-expressing cells and relationship to circadian clock cells in central brain. Dev. Biol. 178: 418-429.

Saunders, D.S. 1966. Larval diapause of maternal origin-II. The effect of photoperiod and temperature on Nasonia vitripennis. J. Insect Physiol. 12: 569-581.

Saunders, D.S. 1971. The temperature-compensated photoperiodic clock 'programming' development and pupal diapause in the flesh-fly, Sarcophaga argyrostoma. J. Insect Physiol. 17: 801-812.

Saunders, D.S. 2002. Insect Clocks. 3rd edn. Elsevier Science, Amsterdam.

Saunders, D.S. 2009. Circadian rhythms and the evolution of photoperiodic timing in insects. Physiol. Entomol. 34: 301-308.

Saunders, D.S. 2012. Insect photoperiodism: seeing the light. Physiol. Entomol. 37: 207-218.

Saunders, D.S. and R.C. Bertossa. 2011. Deciphering time measurement: the role of circadian 'clock' genes and formal experimentation in insect photoperiodism. J. Insect Physiol. 57: 557-566. 


\section{Review Copy - Not for Redistribution Shinsuke Goto - Osaka City University - 10/17/17}

Saunders, D.S. and B. Cymborowski. 1996. Removal of optic lobes of adult blow flies Calliphora vicina leaves photoperiodic induction of larval diapause intact. J. Insect Physiol. 42: 807-811.

Saunders, D.S., V.C. Henrich and L.I. Gilbert. 1989. Induction of diapause in Drosophila melanogaster: Photoperiodic regulation and the impact of arrhythmic clock mutations on time measurement. Proc. Natl. Acad. Sci. USA 86: 3748-3752.

Schmidt, P.S., C.-T. Zhu, J. Das, M. Batavia, L. Yang and W.F. Eanes. 2008. An amino acid polymorphism in the couch potato gene forms the basis for climatic adaptation in Drosophila melanogaster. Proc. Natl. Acad. Sci. USA 105: 16207-16211.

Shiga, S. and H. Numata. 1997. Induction of reproductive diapause via perception of photoperiod through the compound eyes in the adult blow fly, Protophormia terraenovae. J. Comp. Physiol. A 181: 35-40.

Shiga, S. and H. Numata. 2000. The roles of neurosecretory neurons in the pars intercerebralis and pars lateralis in reproductive diapause of the blow fly, Protophormia terraenovae. Naturwissenschaften 87: 125-128.

Shiga, S. and H. Numata. 2009. Roles of PER-immunoreactive neurons in the circadian rhythm and photoperiodism in the blow fly, Protophormia terraenovae. J. Exp. Biol. 212: 867-877.

Shiga, S., N.T. Davis and J.G. Hildebrand. 2003. Role of neurosecretory cells in the photoperiodic induction of pupal diapause of the tobacco hornworm Manduca sexta. J. Comp. Neurol. 462: 275-285.

Shimizu, I., Y. Yamanaka, Y. Shimazaki and T. Iwasa. 2001. Molecular cloning of Bombyx cerebral opsin (boceropsin) and cellular localization of the expression in the silkworm brain. Biochem. Biophys. Res. Commun. 287: 37-34.

Stanewsky, R., M. Kaneko, P. Emery, B. Beretta, K. Wager-Smith, S.A. Kay, M. Rosbash and J.C. Hall. 1998. The $\mathrm{cry}^{b}$ mutation identifies cryptochrome as a circadian photoreceptor in Drosophila. Cell 95: 681-692.

Steel, C.G.H. and A.D. Lees. 1977. The role of neurosecretion in the photoperiodic control of polymorphism in the aphid Megoura viciae. J. Exp. Biol. 67: 117-135.

Stengl, M. and U. Homberg. 1994. Pigment-dispersing hormone-immunoreactive neurons in the cockroach Leucophaea maderae share properties with circadian pacemaker neurons. J. Comp. Physiol. A 175: 203-213.

Tagaya, J., H. Numata and S.G. Goto. 2010. Sexual difference in the photoperiodic induction of pupal diapause in the flesh fly Sarcophaga similis. Entomol. Sci. 13: 311-319.

Tamaki, S., S. Takemoto, O. Uryu, Y. Kamae and K. Tomioka. 2013. Opsins are involved in nymphal photoperiodic responses in the cricket Modicogryllus siamensis. Physiol. Entomol. 38: 163-172.

Tauber, E., M. Zordan, F. Sandrelli, M. Pegoraro, N. Osterwalder, C. Breda, A. Daga, A. Selmin, K. Monger, C. Benna, E. Rosato, C.P. Kyriocou and R. Costa. 2007. Natural selection favors a newly derived timeless allele in Drosophila melanogaster. Science 316: 1895-1898.

Tauber, M.J., C.A. Tauber and S. Masaki. 1986. Seasonal Adaptations of Insects. Oxford University Press, New York.

Tomioka, K. and A. Matsumoto. 2010. A comparative view of insect circadian clock systems. Cell. Mol. Life Sci. 67: 1397-1406.

Tomioka, K., O. Uryu, Y. Kamae, Y. Umezaki and T. Yoshii. 2012. Peripheral circadian rhythms and their regulatory mechanism in insects and some other arthropods: a review. J. Comp. Physiol. B 182: 729-740.

Tyshchenko, V.P. 1966. Two-oscillatory model of the physiological mechanism of insect photoperiodic reaction. Zh. Obshch. Biol. 27: 209-222 (In Russian).

Uryu, M., Y. Ninomiya, T. Yokoi, S. Tsuzuki and Y. Hayakawa. 2003. Enhanced expression of genes in the brains of larvae of Mamestra brassicae (Lepidoptera: Noctuidae) exposed to short day length or fed Dopa. Eur. J. Entomol. 100: 245-250. 


\section{Insect Molecular Biology and Ecology}

Vafopoulou, X. and C.G.H. Steel. 2012. Metamorphosis of a clock: remodeling of the circadian timing system in the brain of Rhodnius prolixus (Hemiptera) during larval-adult development. J. Comp. Neurol. 520: 1146-1164.

Vafopoulou, X., K.L. Terry and C.G.H. Steel. 2010. The circadian timing system in the brain of the fifth larval instar of Rhodnius prolixus (Hemiptera). J. Comp. Neurol. 518: 1264-1282.

Vaz Nunes, M. and J. Hardie. 1993. Circadian rhythmicity is involved in photoperiodic time measurement in the aphid Megoura viciae. Experientia 49: 711-713.

Vaz Nunes, M. and D.S. Saunders. 1999. Photoperiodic time measurement in insects: a review of clock models. J. Biol. Rhythms 14: 84-104.

Veerman, A. 2001. Photoperiodic time measurement in insects and mites: a critical evaluation of the oscillator-clock hypothesis. J. Insect Physiol. 47: 1097-1109.

Wang, Q., Y. Egi, M. Takeda, K. Oishi and K. Sakamoto. 2014. Melatonin pathway transmits information to terminate pupal diapause in the Chinese oak silkmoth, Antheraea pernyi, and through reciprocated inhibition of dopamine pathway functions as a photoperiodic counter. Entomol. Sci. DOI: 10.1111/ens.12083.

Wise, S., N.T. Davis, E. Tyndale, J. Noveral, M.G. Folwell, V. Bedian and K.K. Siwicki. 2002. Neuroanatomical studies of period gene expression in the hawkmoth, Manduca sexta. J. Comp. Neurol. 447: 366-380.

Williams, K.D., M. Busto, M.L. Suster, A.K.C. So, Y. Ben-Shahar, S.J. Leevers and M.B. Sokolowski. 2006. Natural variation in Drosophila melanogaster diapause due to the insulin-regulated PI3-kinase. Proc. Natl. Acad. Sci. USA 103: 15911-15915.

Yuan, Q., D. Metterville, A.D. Briscoe and S.M. Reppert. 2007. Insect cryptochromes: Gene duplication and loss define diverse ways to construct insect circadian clocks. Mol. Biol. Evol. 24: 948-955.

Zhu, H., I. Sauman, Q. Yuan, A. Casselman, M. Emery-Le, P. Emery and S.M. Reppert. 2008. Cryptochromes define a novel circadian clock mechanism in monarch butterflies that may underlie sun compass navigation. PLoS Biol. 6: E4. 